\title{
ASSESSMENT OF MANDIBULAR ASCENDING RAMUS CORTICAL PLATES FOR RECONSTRUCTION OF ATROPHIC MAXILLARY RIDGES VERSUS CHIN CORTICAL PLATES [A RANDOMIZED CLINICAL TRIAL]
}

Ahmed Yousri Zayed*, Nevien Abd Elatif Askar** and Mohamed Atef Abdel Rasoul ${ }^{* * *}$

\begin{abstract}
Aim of the study: Assessment of the quality and quantity of horizontal bone gain upon using the rami cortical blocks versus chin blocks for horizontal augmentation of atrophic maxillary ridges

Patients and Methods: Twenty operation sites in 18 patients suffering from partial edentulous ridge in the maxilla with an inadequate bone width to allow favorable implant placement were selected from the outpatient clinic of Oral and Maxillofacial Surgery Department, Faculty of Dentistry, Cairo University. They were classified after being included in the study into (10 chin group candidate) \& (10 ascending ramus candidate).
\end{abstract}

Results: A total of 20 atrophic sites (cases) in 18 patients ranging from 29-54 years old with a mean of 43.4 years were included in the present study (Table 2). The enrolled subjects were randomly divided by alternation into two groups. 10 sites received autogenous block graft harvested from the mandibular symphysis (symphyseal group). Other 10 sites received autogenous block graft harvested from the ascending ramus (ramus group).

Conclusion: Within the limits of this study the following could be concluded:

Ascending ramus onlay blocks showed significant better results than symphyseal onlay blocks regarding both the quality and stability of the grafted volume of bone.

Ramus graft harvesting is a more sensitive procedure as compared to symphyseal block graft and requires certain level of surgical skill and experience to be performed successfully.

\footnotetext{
* Master Degree in Dental Implantology

** Professor of Oral and Maxillofacial Surgery Cairo University.

*** Lecturer of Oral and Maxillofacial Surgery Cairo University
} 


\section{INTRODUCTION}

There are different causes for maxillary ridge atrophy such as dental loss, trauma, infection, bone pathology and may be physiological atrophy. Atrophied maxilla considers a serious limitation for implant placement. This makes a challenge to the surgeon for implant placement in relation to the planned prosthetic positions (Ali 1 et al., 2014).

Implant rehabilitation has presented higher success rates of 84-92\%, when sufficient amount of bone is available in maxilla. But atrophy in maxilla is a common finding in the form of centripetal pattern of alveolar resorption, presence of nasal fossae, pneumatization of maxillary sinuses and poor bone quality and quantity complicate the implant placement (Marco 2 et al., 2005)

There are many solutions for dealing with this problem as horizontal and vertical ridge augmentation and sinus bone graft. Socket preservation is a method of prevention of this problem (Thanakone 3, 2014)

Atrophic ridge augmentation can be done using variety of bone grafts which can be autogenous or non-autogenous or synthetic materials; in autogenous grafts; bone is transferred from donor site to recipient site in the same individual, it can be cortical or cancellous or a combination of both and is characterized by no immunogenic rejection. They can be obtained from the mandible, maxilla, tibia, iliac crest and cranium. Although they have osteoinductive, osteogenic and osteoconductive properties, they have their drawbacks like additional surgical procedures, treatment delay and morbidity at donor sites (Harry 4 et al., 2012; Precheur 5, 2007).

But in allografts (homologous, homograft's); bone grafts have been taken from different individual of the same species. As fresh or fresh frozen bone closely matches the recipient site in constitutional elements demineralized freeze-dried bone allografts (DFDBA) and mineralized freeze-dried allografts (FDBA) (Bellini and Romeo6 et al., 2009).
Allograft has a little osteo-conductive and osteo-inductive capability because of the low concentration of bone growth proteins for removal of potential antigenicity and pathogenicity. Also its draw backs include antigenicity and transmission of bone disease (Avinash and Khalid 7, 2013).

But xenografts; materials taken from different species (bovine bone), show less resorption of graft substrate and form less new bone during the first few months, reduced operative time and no morbidity at the donor site. Disadvantages as antigenicity and infectious disease transmission are present.

In alloplastic type; grafts derived from inert synthetic materials like Hydroxyapatite (HA), calcium phosphate, b-tri calcium phosphate Calcium sulfate (gypsum), bioactive glasses, poly methyl methacrylate (Bertil 8, 2008).

There was increased resorption time and decreased new bone formation as no cellular or protein material within these grafts. Growth factors as recombinant platelet-rich plasma (PRP), Plateletderived growth factors (PDGF), Transforming growth factor (TGF-b), and Bone morphogenetic protein (BMP) can be added to all the above graft materials to enhance bone formation and reduce the healing time (Chiriac 9 et al., 2005; Enneking 10 et al., 1980). The use of graft material should be chosen in relation to each need i.e., type, size, and location of defect; loco-regional conditions; type of surgical procedure; material properties (Aghaloo and Moy 11, 2007).

Autogenous bone grafts remain the gold standard for bone grafting due to its all properties. However, when the autogenous grafts transferred to a site has the same origin of developmental bone gives better results so the mandibular bone either from the ramus or the symphysis is the ideal choice for ridge augmentation of atrophic ridges (Menini 12 et al., 2012; Clavero and Lundgren 13, 2003).

The intra membranous mandibular bone grafts give less resorption with better incorporation than 
endochondral bone grafts (iliac bone). Researchers presented a three dimensional (3D) reconstruction technique for atrophic ridges and complicated vertical bone defects using mandibular bone block graft "the gold standard". There are several possibilities for augmentation of bone volume depending on situation, indication and adequate diagnosis; the treatment options can be extended from minimally invasive procedures with locally harvested bone grafts in local anesthesia, to very sophisticated grafting techniques for $3 \mathrm{D}$ bone reconstruction with extra oral harvested bone grafts. Khoury reported that inhis cases ascending ramus grafts is almost 5 times more than chin grafts (Urban 14, 2017; Khoury 15et al., 2007).

In the present study, Khoury techniques were used in autogenous bone harvesting of bony defects of atrophic maxillary ridges for assessment of ascending ramus cortical plates versus chin cortical plates. As this techniques use block grafts of cortical plates for $2 \mathrm{D}$ or $3 \mathrm{D}$ reconstructions. So it would be more accurate and easy to assess cortical plates from the ascending ramus and the chin.

\section{Aim of the study:}

Assessment of the quality and quantity of horizontal bone gain upon using the rami cortical blocks versus chin blocks for horizontal augmentation of atrophic maxillary ridges.

\section{PATIENTS AND METHODS}

\section{A. Patients}

Twenty operation sites in 18 patients suffering from partial edentulous in the maxilla with an inadequate bone width to allow favorable implant placement were selected from the outpatient clinic of Oral and Maxillofacial Surgery Department, Faculty of Dentistry, Cairo University. They were classified after being included in the study into (10 chin group candidate) \& (10 ascending ramus candidate)

\section{Inclusion criteria:}

Patients with atrophic maxilla with residual alveolar bone height not less than $10 \mathrm{~mm}$.

Alveolar bone width from $1-4 \mathrm{~mm}$

Both sexes.

No intraoral soft and hard tissue pathology

No systemic condition that contraindicate implant placement

\section{Exclusion criteria:}

Bone pathology.

Smokers (heavy)

Patients with systemic disease that may affect normal healing (hyperparathyroidism, patients under radio therapy and chemotherapy).

Patients with Para functional habits.

The present study was approved by the ethics committee of the Faculty of Dentistry, Cairo University.

Preoperative Assessment: A preoperative assessment for all enrolled candidates was carried out including history taking, clinical and radiographic examination.

\section{Patient Interview:}

Each patient was interviewed in order to obtain a comprehensive history including a full medical and dental history. The purpose, the nature of this study and detailed surgical procedure with possible complications were discussed with the patients and a written consent was signed.

\section{Clinical Examination:}

Patients were inspected for adequate Inter- arch space, normal covering mucosa and periodontal status of adjacent teeth.

The ridge was palpated to check the contour for any abnormalities that may interfere with implant placement. 
In this study only FB1, FB2\&FB3 prosthetic options were clinically selected to be enrolled according to Misch16 et al., in 2005.

\section{Radiographic Examination:}

A preoperative digital panoramic radiograph with 1:1 magnification was taken for each patient as a primary survey to identify the deficient areas and assess the presence of any remaining roots or pathological lesions at the area of interest. (Figure 2)

For the selected patients a $\mathrm{CBCT}(*)($ diagnostic for upper and lower arch pre- operatively) was ordered.

All patients were instructed to close their mouths in a centric occlusion during taking all CBCT scans in the study (preoperatively, immediate postoperatively and 6 months post- operatively). Then, when drawing the panoramic curve on the axial view of the software, the guiding points of the line which determine the shape of the panoramic curve should be put on the center of natural teeth and / or the center of the edentulous ridge. While, the horizontal coordinate of the panoramic view was on the crest of the alveolar native bone. So, the opposing teeth, foramina and sinus or nasal septa could be taken as anatomical landmark (reference points) for repeated cuts pre-operatively, immediate post-operatively and 6 months post-operatively.

Pre-operative CBCT scan was ordered for the enrolled candidates to:

\section{A- At recipient sites:}

1. Assess the extent of the defect

2. Evaluate ascending rami and the symphyseal donor sites.

3. Accurate measurements of the residual bone height and width at the atrophic sites

\section{B-At donor sites :}

For ascending ramus:

The position of inferior alveolar canal was detected
The thickness of the cortical block of bone was checked.

\section{For symphysis:}

The mental nerves, loops and foramina were measured.

The roots of natural lower incisors were detected.

The thickness of the cortical blocks and the underling spongy bone were detected.

A scout view was obtained and adjustments were made to ensure that the all patients were correctly aligned in the scanner according to the adjustment of light beam before acquisition.

The available height and width at each edentulous site should be measured accurately in the reformatted cross sectional images. The alveolar height was measured form the alveolar crest to the nasal floor or maxillary sinus floor.

The alveolar width was measured $2 \mathrm{~mm}$ below the crest (the line of

The patients were randomly divided using a computer generated random numbers were used to divide patients into two groups: ramus group and symphyseal group.

\section{Surgical Procedure:}

First stage surgery: for bone augmentation

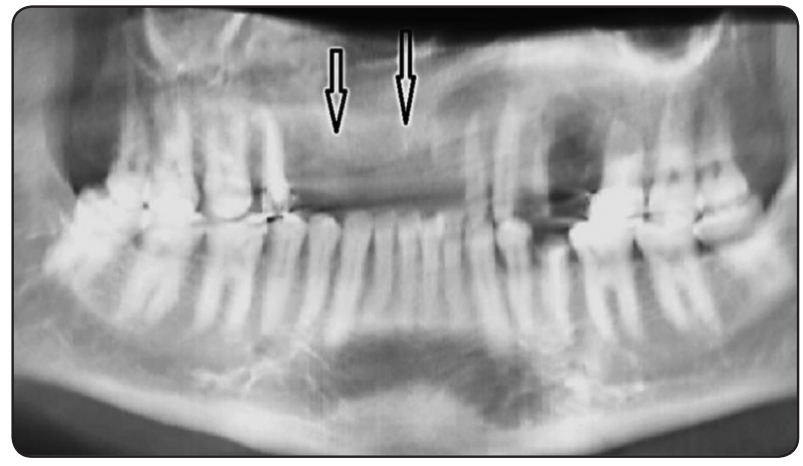

Fig. (1) Pre-operative reformatted panoramic view of CBCT (patient biting in centric occlusion) showing site of the alveolar defect "Case No.10". 

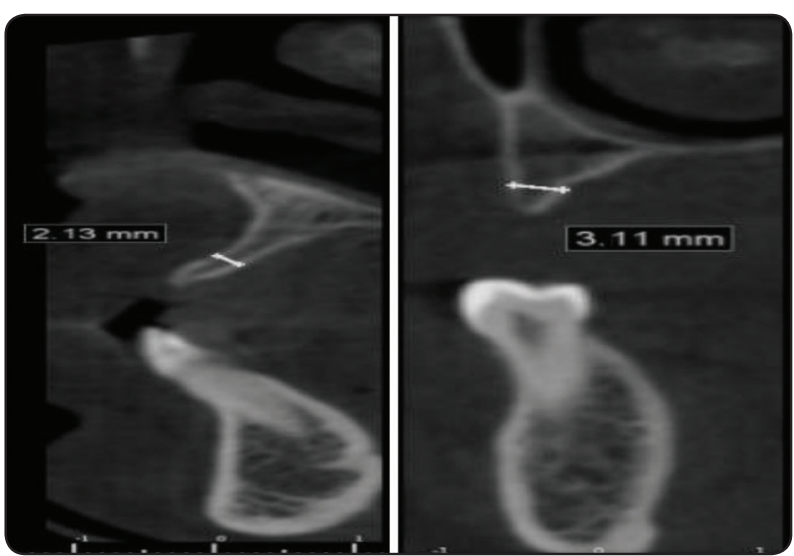

Fig. (2) Preoperative reformatted cross sectional CBCT cuts showing calculation of the horizontal bone dimensions of the ridge 1: chin group; 2 ramus group "Case No.9"

Second stage surgery: for removal of fixating screws and implants placements

\section{First stage surgery (bone augmentation)}

All cases were operated under conscious sedation. In conscious sedation method the patient maintains an adequate level of consciousness so that the patient remains responsive to physical stimulation or verbal commands during the procedure and retains the protective reflexes and the ability to breathe without external assistance. The type of sedation used depends on several factors as patient's medical history and level of anxiety, in combination with the severity and length of the necessary dental treatments. Patients were asked to rinse their mouths with Chlorhexidine HCL $1.25 \%$ mouthwash immediately preoperative. All procedures were performed under local anesthesia using [Articaine, 4\% 1:100000 epinephrine].

- Initially, the recipient site was adequately exposed using a mid Crestal and sulcular incision extending a minimal of one tooth on each side of the defect and two vertical releasing incisions. Carefully, a three line full pyramidal mucoperiosteal flap was elevated and reflected to expose the underlying bone without leaving any soft tissue fragment on the bone, minimal reflection of the palatal mucoperiostum.

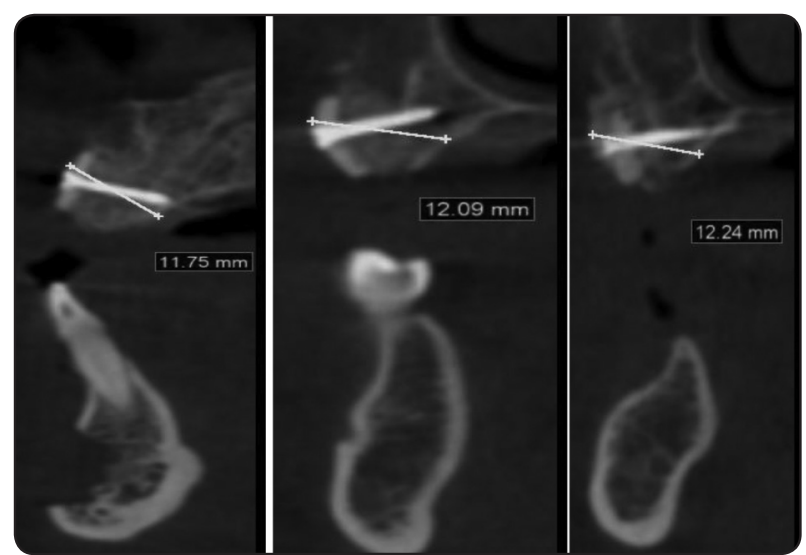

Fig. (3) Immediate post-operative CBCT A: chin cortical plate of bone (symphyseal group) B \& C: ascending ramus cortical plate of bone (ramus group). "Case No.9

\section{Ramus graft harvesting}

Khoury 17 et al., in 2015, A trapeze-like incision (starting distal to the second molar with a 2-cm vestibular incision over the ramus bone, continuing parallel and lateral to the second molar, and then going back in the vestibular direction on the distal border of the first molar) was followed by the elevation of a mucoperiosteal flap (similar to that used for the removal of impacted third molars). This exposed the bone at the level of the external oblique ridge to a length of 3 to $4 \mathrm{~cm}$ and a depth of $2 \mathrm{~cm}$ (Figure 3). The volume of bone to be harvested depended on the size and extent of the external oblique ridge and the quantity of bone needed for grafting

The harvesting osteotomy was performed with the MicroSaw following a clear protocol. The MicroSaw consists of an 8-mm-diameter, 0.25-mmwide diamond disk mounted on a contra-angle or MicroSaw hand piece with a soft tissue protector. In the harvesting protocol, three osteotomies were performed with the diamond disk: two proximo-vertical osteotomies were made with the MicroSaw hand piece, and one was made apicohorizontally with the contra-angle hand piece. The apico-horizontal osteotomy was made slightly overlapping both vertical osteotomies basally. Once the osteotomy lines were positioned apical to the 
alveolar nerve level, the MicroSaw's maximum cutting capacity of $3.2 \mathrm{~mm}$ was avoided in the distal section the donor site, starting directly behind the second molar. In this area, the maximal depth of the incision with the diamond disk was $2 \mathrm{~mm}$ (the diamond layer is $1 \mathrm{~mm}$ wide). ${ }^{(15)}$

The final osteotomy, a fourth Crestal (superior) complete cut was done by using the MicroSaw connecting the two vertical cuts on the crest of the bone parallel to the external oblique ridge and parallel to the buccal wall of the ramus (approximately 4 to $5 \mathrm{~mm}$ medial to the external border of the external oblique line) and between the two vertical incisions leading to easy lateral dislocation of the bone block (Figure 7). The donor site was typically sealed with collagen fleece after smoothening (beveling) of sharp lines of osteotomies with a small fissure bur.

\section{Symphyseal Graft Harvesting}

The borders of the osteotomy were at least $5 \mathrm{~mm}$ away from the teeth apices, inferior border of the mandible and mental foramina.

Incision Design: Pikos and Michael 18, in 2005, utilized two approaches to expose the symphysis.

A) The sulcular approach: This approach was chosen in cases where the periodontium was healthy and no crowns were present in the anterior dentition that could present aesthetic problems with associated gingival recession, or when the anterior region was the defect site. The incision begins in the gingival sulcus from second bicuspid of one side to second bicuspid of the other side. An oblique releasing incision was made at the distal buccal line angle of these teeth and continues into the depth of the buccal vestibule. A full thickness mucoperiosteal flap was reflected down to the inferior border, (de gloving of the mandibular symphysis). The mental neurovascular bundles were identified bilaterally.

B) The vestibular design: This approach was indicated in cases with compromised periodontium in the lower anterior region, when crown margins of the mandibular anterior teeth were within the aesthetic or speaking zones, as gingival recession may cause their exposure. The flap began with a beveled partial- to full-thickness mucoperiosteal incision. The incision was placed $5-10 \mathrm{~mm}$ below the muco-gingival junction, extending just distal to the mandibular canines to allow for adequate access and easier adaptation of the flap for tensionfree closure. The flap was carefully elevated to expose the symphysis region Osteotomy design was performed using MicroSaw disk. The graft size should be $2 \mathrm{~mm}$ larger than the defect size to allow for graft contouring.

The osteotomies were completed and connected together using straight bi-beveled chisels and mallet

Schettler 19, D, in 1976, reported that elevation of the graft was started using a bi-beveled chisel and mallet. In case of unilateral block harvest or two separate bilateral block harvest, elevation started from the medial cut (towards the midline) In case of a single bilateral block harvest, elevation of the block should start from the superior end of the vertical cuts. This is to prevent any unnecessary trauma to the mental neurovascular bundle .

\section{WOUND CLOSURE}

The ascending ramus donor sites were sutured with 4/0 synthetic monofilament multiple simple interrupted sutures(*). Moreover, the mandibular symphysis donor site was closed in 2 layers, the deep muscular layer was first closed by multiple horizontal mattresses using 4/0 vicryl resorbable sutures(**). The mucosal layer was then closed with continuous with lock 4/0 synthetic monofilament suture. For the grafted sites in both groups, buccal flap advancement was done by the periosteo-elastic technique which is mostly bladeless by gentle periosteal incision at the line connecting the two vertical incisions, then cutting of the subperiosteal 
bundles and separation of elastic fibers with a blunt periosteal instrument in a coronal pushing motion urban 14 , in 2017. It was performed to release the periosteal tissue and allow for tension free closure of the flap. Closure was done using apical horizontal mattress and crestal simple interrupted sutures (two layers) with 4/0 synthetic monofilament sutures

\section{Postoperative care and follow up:}

- All patients received post -operative antibiotic (Amoxicillin/Clavulanic acid(*)) 1gram every 12 hours orally for 5 days, and non-steriodal anti- inflammatory analgesic (Ibuprofen $(* *)$ ) $600 \mathrm{mg}$ every 8 hours orally for 5 days.

- Patients were instructed to follow oral hygiene measures and to use chlorohexidine $0.2 \%(* * *)$ mouthwash for 2 weeks.

- Sutures were removed two weeks postoperative.

- All patients were clinically evaluated at 1 week, 2 weeks, 1 month and 6 months post operatively.

\section{Post-operative Assessment:}

\section{Neurosensory assessment:}

Neurosensory evaluation of the inferior alveolar nerve by function questionnaire to indicate any alterations in sensation

- The patients were asked to fill up the subjective assessment questionnaire at the area of lower lip and chin region, which consists of absence of sensation b. altered sensation c. numbness.

- Objective test using light touch, brush stroke direction and two- point discrimination tests after patients being seated comfortably relaxed and closed eyes.

- Patients' descriptions of sensations were recorded in Medical Research

Council Scale (MRC) scale (Park and Indersano 20 2014).

\section{Medical Research Council Scale}

\begin{tabular}{|l|l|}
\hline Grade & Description \\
\hline S0 & No sensation \\
\hline S1 & Deep cutaneous pain in an autonomous zone \\
\hline S2 & Some superficial pain and touch sensation \\
\hline S2+ & $\begin{array}{l}\text { Superficial pain and touch sensation plus } \\
\text { hyperesthesia }\end{array}$ \\
\hline S3 & $\begin{array}{l}\text { Superficial pain and touch sensation without } \\
\text { 15 meresthesia; static 2- point discrimination }>\end{array}$ \\
\hline S3+ & $\begin{array}{l}\text { Same as S3 with good stimulus localization } \\
\text { and static 2-point discrimination of 7-15 mm }\end{array}$ \\
\hline S4 & $\begin{array}{l}\text { Same as S3 and static 2-point } \\
\text { discrimination of 2-6 mm }\end{array}$ \\
\hline
\end{tabular}

\section{Radiographic assessment}

Immediate post-operative CBCT scans were ordered for all patients and at six month interval, for accurate measurement of bone width to assess the amount of horizontal bone gain of the grafted ridges.

After acquisition, every scan was adjusted to reproduce the same pre- operative view to ensure accurate measurements

All data were collected and tabulated for statistical analysis

Immediate post-operative CBCT A: chin cortical plate of bone (symphyseal group) B \& C: ascending ramus cortical plate of bone (ramus group). "Case No.9

\section{Histomorphometric analysis method:}

At the time of implant installation, core biopsies were taken. The specimens were immediately fixed in $10 \%$ buffered formalin for one week, after that decalcified and processed according to standardized protocol ethylene dimene-tetra acetate (EDTA) / formic acid combination, then specimens were embedded longitudinally into paraffin blocks and 
oriented in a standardized way for labeling and differentiating the newly formed bone end from the native bone end. Blocks were cut into longitudinal 5 $\mu \mathrm{m} /$ thick sections using a manual rotary microtome (RM 2135 microtome, Leica, Heidel Berger StraBe, Nussloch, Germsany), and stained with Mayer's hematoxylin and eosin stain (H\&E) for

\section{Histomorphometric analysis.}

Only three slides for every biopsy were used to assess the histomorphometric results and mean value was taken for every biopsy and tabulated for statistical analysis

\section{Radiographic assessment}

For all preoperative, immediate post-operative and 6 months post-operative CBCTs:

Were taken while the patients were biting in centric occlusion.

The panoramic curves points were located on the center of the crest of the ridge and/or natural teeth.

All cuts which were chosen for measurements were correlated by anatomical landmarks (e.g tooth, foramen, sinus septum and nasal septum) to be easily repeated.

All radiographic measurements (5 measurements for every site) were accurately taken. The mean of measurements of every site were tabulated for statistical analysis

\section{Statistical Analysis}

Data management and statistical analysis were performed using the Statistical Package for Social Sciences (SPSS) version 22. Numerical data were summarized using means and standard deviations. Data were explored for normality using KolmogrovSmirnov test and Shapiro-Wilk test. Comparisons between 2 groups and overtime were done by 2 way repeat ed measures ANOVA. Comparisons between the 2 groups at each time point were done using the independent t-test. Overtime comparisons in each group were done by repeated measure ANOVA followed by Post hoc paired t-test. All p-values are two-sided. P-values $\leq 0.05$ were considered significant.

\section{RESULTS}

A total of 20 atrophic sites (cases) in 18 patients ranging from 29-54 years old with a mean of 43.4 years were included in the present study (Table 2). The enrolled subjects were randomly divided by alternation into two groups. 10 sites received autogenous block graft harvested from the mandibular symphysis (symphyseal group). Other 10 sites received autogenous block graft harvested from the ascending ramus (ramus group).

TABLE (2) Demographic data and the defect site of symphyseal group patients.

\begin{tabular}{|c|c|c|c|}
\hline $\begin{array}{c}\text { Case no. } \\
\text { site }\end{array}$ & Age & Sex & Maxillary defect \\
\hline 10 & 42 & M & L1,2\& R1,2,3 \\
\hline 11 & 39 & F & L1,2,3 \\
\hline 9 & 35 & F & L1,2\& R4,5,6,7 \\
\hline 12 & 29 & M 1 \\
\hline 13 & 36 & M & R1,2,3 \\
\hline 14 & 43 & F & L 1,2 \\
\hline 15 & 46 & M & L1\&R1 \\
\hline 16 & 40 & F & R1,2,3,4 \\
\hline 17 & 47 & M & L1,2,3\& R1,2,3 \\
\hline 18 & 53 & M & L1,2,3 \&R1 \\
\hline
\end{tabular}

$R:$ right $\quad$ L : left 
TABLE (3) Demographic data and the defect site of ascending ramus group patients.

\begin{tabular}{|c|c|c|c|}
\hline Case no. site & Age & Sex & Maxillary defect \\
\hline 1 & 41 & M & R3,4,5 \\
\hline 2 & 43 & F & L5,6,7 \\
\hline 3 & 51 & F & R4,5,6 \&L 4,5,6 \\
\hline 3 & 51 & F & R4,5,6 \&L 4,5,6 \\
\hline 4 & 45 & F & R2,3,4 \\
\hline 5 & 43 & M & R4,5,6,7 \\
\hline 6 & 54 & M & R4,5 \\
\hline 7 & 34 & F & L1,2\&R1,2,3 \\
\hline 8 & 47 & M & L4,5,6\&R4,5,6 \\
\hline 9 & 35 & F & L1,2 \&R4,5,6,7 \\
\hline
\end{tabular}

$R:$ right $\quad L:$ left

\section{Clinical Results:}

\section{Ramus Group:}

All patients showed uneventful soft tissue healing with no infection, sequestration of the grafted cortical plate, or neurosensory deficits.

According to MRC scale, all patients recorded 2- point discrimination of 2-6 $\mathrm{mm}$ (S4) only after two weeks postoperatively.

At 6 months during re-entry, all cases revealed sufficient bone width that allowed conventional implant placement in all cases, without any further augmentation procedures

\section{Symphyseal group:}

All patients healed uneventfully, no signs of infection or wound dehiscence was noted. Post operative swelling was still noted at suture removal 2 weeks post operatively specially at the donor sites.
According to MRC scale, two patients recorded slight altered sensation (S2) after one month only one patient of the two recorded superficial pain and touch sensation without hyperesthesia (S3). All patients 3 months postoperatively recorded 2-point discrimination of 2-6 $\mathrm{mm}$ (S4).

\section{Radiographic observations:}

- In all cases of both groups, the graft fixing screws were seen extending bicortically and flushing with the lingual cortex.

- In all cases, of both groups, the vertical level of the graft was at the same level of that of the native alveolar bone.

- In all cases, in both groups, the graft appeared well adapted to the native bone and at 6 months post-operative CBCT the buccal cortex of the grafted atrophied ridge couldn't still be demarcated within the bone, which indicated the union and fusion between the graft and the atrophic ridge.

\section{* Statistical analysis of radiographic measurements}

I- Bone width at different intervals in each group

- Preoperatively, the mean bone width of ramus group was $2.75 \pm 1.5 \mathrm{~mm}$ and increased to a mean of $10.54 \pm 1.2 \mathrm{~mm}$ immediately postoperative (ascending ramus cortical plate augmentation). At 6 months postoperatively, it decreased slightly to reach a mean of $9.86 \pm 1.1 \mathrm{~mm}(\mathrm{p}<0.05)$

TABLE (4) Mean, SD and repeated measure ANOVA test of bone width in ramus group

\begin{tabular}{|c|c|c|c|c|c|c|c|}
\hline & \multicolumn{2}{|c|}{ Preoperative } & \multicolumn{2}{|c|}{ Immediate } & \multicolumn{2}{|c|}{ Postoperative } & \\
\hline & Mean & S.D & mean & S.D & mean & S.D & P-value \\
\hline $\begin{array}{c}\text { Ramus } \\
\text { group }\end{array}$ & 2.75 & 1.5 & 10.54 & 1.2 & 9.86 & 1.1 & $\begin{array}{c}(0.05 \\
(0.097)\end{array}$ \\
\hline
\end{tabular}

$P \leq 0.05$ is considered statistically significant 


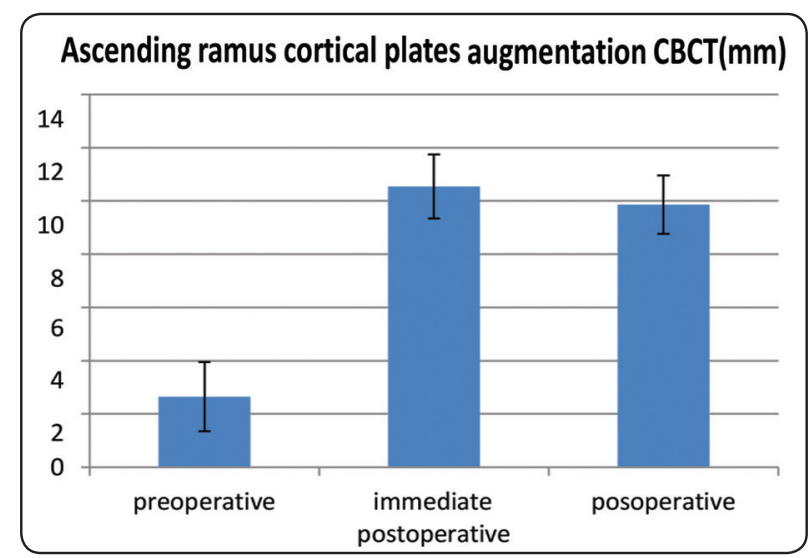

Fig. (4): Mean, SD and repeated measure ANOVA test of bone width in ramus group.

For the symphyseal group, preoperatively the mean bone width of symphyseal group was $2.65 \pm 1.3 \mathrm{~mm}$ and increased to $10.41 \pm 1.79 \mathrm{~mm}$ immediately postoperative. 6 months postoperative, it decreased slightly to reach $8.38 \pm 1.57 \mathrm{~mm}$. This was statistically significant $(\mathrm{p}<0.001)$.

TABLE (5) Mean, SD and repeated measure ANOVA test of bone width in symphyseal group

\begin{tabular}{|l|l|l|l|l|l|l|l|}
\hline & \multicolumn{2}{|l|}{ Preoperative } & \multicolumn{2}{l|}{ Immediate } & \multicolumn{2}{l|}{ Postoperative } & P- \\
\cline { 2 - 7 } & mean & S.D & mean & S.D & Mean & S.D & value \\
\hline $\begin{array}{l}\text { Symphyseal } \\
\text { group }\end{array}$ & 2.65 & 1.3 & 10.41 & 1.79 & 8.38 & 1.57 & $<0.001$ \\
\hline
\end{tabular}

$P \leq 0.05$ is considered statistically significant

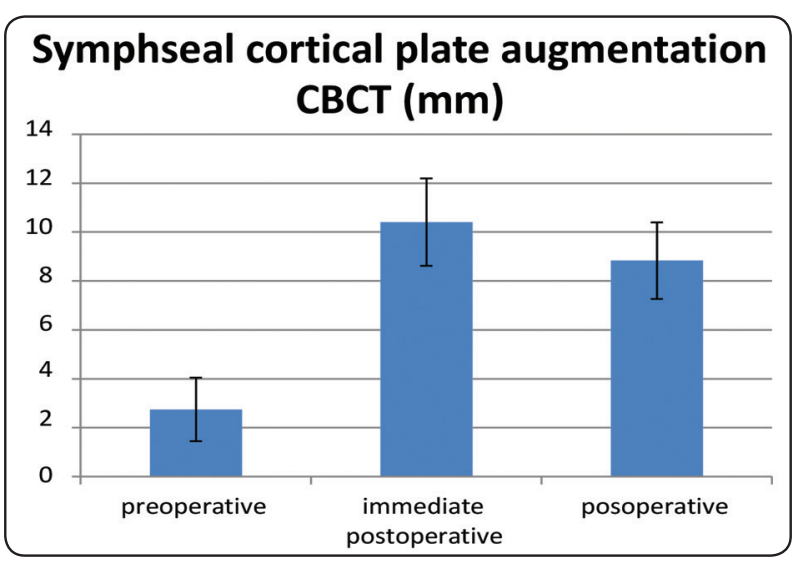

Fig. (5) Mean, SD and repeated measure ANOVA test of bone width in symphyseal group.

\section{II- Comparisons between 2 groups in bone width}

- Preoperative: the mean bone width of ramus group was $2.65 \pm 1.3 \mathrm{~mm}$ compared to $2.75 \pm 1.5$ $\mathrm{mm}$ in symphyseal group. This was statistically insignificant $(\mathrm{p}=0.671)$ (Table 6).

- Immediate Postoperative: the mean bone width of ramus group was $10.54 \pm 1.2 \mathrm{~mm}$ compared to $10.41 \pm 1.79 \mathrm{~mm}$ in symphyseal group. This was statistically significant $(\mathrm{p}<0.05)$.

- 6 months Postoperative: the mean bone width of ramus group was $9.86 \pm 1.1 \mathrm{~mm}$ compared to $8.38 \pm 1.57 \mathrm{~mm}$ in symphyseal group. This was statistically significant $(\mathrm{p}=0.046)$.

TABLE (6) Mean (mm), SD and independent t-test of bone gain in width in ramus and symphyseal groups

\begin{tabular}{|c|c|c|c|c|c|}
\hline & \multicolumn{2}{|l|}{ Ramus group } & \multicolumn{2}{c|}{$\begin{array}{c}\text { symphyseal } \\
\text { group }\end{array}$} & \multicolumn{1}{|c|}{} \\
\hline & Mean & S.D & Mean & S.D & P-value \\
\hline Preoperative & 2.65 & 1.3 & 2.75 & 1.5 & 0.694 \\
\hline Immediate & 10.54 & 1.2 & 10.41 & 1.79 & $\begin{array}{c}<0.05 \\
(0.027)\end{array}$ \\
\hline 6 Months post & 9.86 & 1.1 & 8.38 & 1.57 & 0.002 \\
\hline
\end{tabular}

- $P \leq 0.05$ is considered statistically significant.

\section{III- Comparisons between 2 groups in bone gain}

Bone gain: The mean bone gain of ramus group was $7.21 \pm 0.2 \mathrm{~mm}$ compared to $6.08 \pm 0.07$ in symphyseal group. This was statistically nonsignificant $(\mathrm{p}=0.251)$

Bone gain\%: The mean bone width gain $\%$ of ramus group was $272 \pm 15 \%$ compared to $221.1 \pm$ $4.9 \%$ in symphyseal group. This was statistically non- significant $(\mathrm{p}=0.921)$ 
TABLE (7): Mean, SD and independent t-test of bone gain percentage in ramus and symphyseal groups.

\begin{tabular}{|c|c|c|c|c|c|}
\hline & \multicolumn{2}{|c|}{ Ramus group } & \multicolumn{2}{c|}{$\begin{array}{c}\text { Symphyseal } \\
\text { group }\end{array}$} & \\
\hline & Mean & S.D & Mean & S.D & P-value \\
\hline Bone gain(mm) & 7.21 & 0.2 & 6.08 & 0.07 & 0.251 \\
\hline Bone gain\% & 272 & 15 & 221.1 & 4.9 & 0.921 \\
\hline
\end{tabular}

$P \leq 0.05$ is considered statistically significant

\section{IV- Comparison between 2 groups in graft re- sorption}

- The mean graft resorption of the ramus group was $0.68 \pm 0.1 \mathrm{~mm}$. This was statistically nonsignificant. $(\mathrm{p}=0.08)$

- The mean graft resorption for the symphyseal group was $1.85 \pm 0.22 \mathrm{~mm}$. This was statistically significant. $(\mathrm{p}=0.006)$

- Comparison of the mean graft resorption of ramus group $(0.68 \mathrm{~mm})$ to the symphyseal group $(1.85 \mathrm{~mm})$ was statistically significant. $(\mathrm{p}<0.001)$

TABLE (8) Mean and independent t-test of bone graft resorption in ramus and symphyseal groups.

\begin{tabular}{|c|c|c|c|}
\hline \multirow{2}{*}{} & \multicolumn{2}{|c|}{ Graft resorption } & \multirow{2}{*}{ P-value } \\
\cline { 2 - 3 } & Mean & S.D & \\
\hline Ramus group & 0.68 & 0.2 & $\mathrm{p}=0.08$ \\
\hline Symphyseal group & 1.85 & 0.22 & $\mathrm{p}=0.006$ \\
\hline
\end{tabular}

$P \leq 0.05$ is considered statistically significant.

\section{Histomorphometric}

Although ramus group showed non-significant results of bone quantity according to $\mathrm{CBCT}$, Histomorphometric analysis revealed that bone area $\%$ (bone quality) of ramus group was significantly increased when compared to the corresponding value of symphyseal group

\section{DISCUSSION}

In the present study, 20 surgical sites of maxillary atrophic ridges in eighteen patients were horizontally augmented using autogenous mandibular mono-cortical blocks.10 grafts harvested from the ascending ramus and 10 grafts harvested from mandibular symphysis. These cases were randomly divided into two equal groups. The aim of the study was to compare the quality and quantity of horizontal bone gain upon using the rami cortical blocks versus chin blocks for horizontal augmentation of atrophic maxillary ridges.

Misch 21, in 1997, presented a study to compare the intraoral donor sites for onlay grafting prior to implant placement in fifty patients with horizontally deficient ridge (mandibular bone blocks from the symphysis vs ramus). Dehiscence of the incision occurred in $10.7 \%$ of symphysis donor site patients with vestibular approach. Two patients with symphyseal dehiscence developed infection at the donor site but no infection or dehiscence occurred in ramus graft donor sites. Khoury15 et al in 2007, presented study included 3032 block graft procedures were harvested from the ramus and 549 bone grafts which were harvested from symphysis for reconstruction of atrophic ridges. There were 29 cases (1\%) from ramus group and 15 cases from symphyseal group (2.5\%) suffered from wound dehiscence. ACocella22 et al., in 2010, presented a study in fifteen patients for clinical, histological and Histomorphometric evaluation of the healing of mandibular ramus bone block grafts for alveolar ridge augmentation before implant placement. They had one patient exhibited a graft exposure but the soft tissue dehiscence resolved spontaneously without further complications.

More than that, using fresh frozen tibial human block grafts donor sites, Acocella 23 et al., in 2012, operated eighteen block grafts in sixteen patients with atrophic maxillary ridge and had one patient with early exposure of the block after first stage surgery and required a second surgical procedure to cover it. Chiapasco59 et al, in 2013, there were two 
graft exposure in the 8 patients treated with FFB blocks.

Moreover, our results were better than AlGhamdi24, in 2013, when he studied the postsurgical complications of the symphyseal bone block graft with and without soft tissue grafting in fourteen sites from twelve's patient. AlGhamdi had two patients with soft tissue dehiscence and one of those lost $45 \%$ of the graft and the other $75 \%$ of the graft due to soft tissue dehiscence (the last required regrafting). Lastly, our results were on the contrary to Osman and Atef 25, in 2018, who presented a novel approach for computer guided chin harvest in twenty patients and had three patients suffered from wound dehiscence.

On the contrary to previous studies, in the present study all augmentation procedures in all included patients showed a successful osseointegration and consolidation of the blocks with the recipient sites without complications or wound dehiscence of the donor or recipient sites and were successfully treated with successful dental implants.

This could be attributed to the difference between these studies and our present study was that in our study the grafts were well-smoothened; the flap margins were properly released using periosteoelastic technique which is mostly bladeless by gentle periosteal incision (as basally to the flap as possible) at the line connecting the two vertical incisions, then cutting of the subperiosteal bundles and separation of elastic fibers with a blunt periosteal instrument in a coronal pushing motion till enough elasticity was achieved urban 14, in 2017. It was performed to release the periosteal tissue and allow for tension free closure of the flap.

The closure of the flap (at the recipient sites) was performed in two layers. The first layer is closed with multiple horizontal mattress sutures placed $5 \mathrm{~mm}$ apical to the incision line, and then superimposed with crestal simple interrupted sutures to close the edges of the flap with $4 / 0$ synthetic monofilament sutures.

\section{SUMMARY}

The present study is a randomized clinical trial to evaluate the efficacy of atrophic maxillary ridge augmentation by ramus block graft versus symphyseal block graft.

Eighty patients (with 20 operating sites) seeking implant rehabilitation for the atrophic maxillary ridge were selected from the outpatient clinic of the Oral and Maxillofacial Surgery Department, Faculty of Dentistry, Cairo University. 10 sites were augmented by ramus block graft and the other 10 sites were augmented using symphyseal block graft.

All patients were regularly assessed both clinically and radiographically up to 6 months postoperatively. Histomorphometric analysis was performed using biopsies during implants placement.

Statistical analysis showed that both groups showed a significant increase in alveolar bone width immediately post-operative and at 6 months. The mean graft resorption in the ramus group 6 months post- operative was considered non- significant while in the symphyseal group the mean graft resorption was significant. And the mean graft resorption of ramus group compared to the symphyseal group was statistically significant. Bone area \% of ramus group was significantly increased when compared to the corresponding value of symphyseal group

\section{CONCLUSION}

Thus within the limits of this study the following could be concluded:

Ascending ramus onlay blocks showed significant better results than symphyseal onlay blocks regarding both the quality and stability of the grafted volume of bone.

Ramus graft harvesting is a more sensitive procedure as compared to symphyseal block graft and requires certain level of surgical skill and experience to be performed successfully. 


\section{REFERENCES}

1. Ali S A, Karthigeyan S, Deivanai M and Kumar A: Implant Rehabilitation For Atrophic Maxilla: A Review J Indian Prosthodont Soc (2014) 14(3):196-207.

2. Marco S, Juan $\mathrm{G}$ et al : Implant rehabilitation of the atrophic upper jaw Med Oral Patol Oral Cir Bucal (2005)10: E45-E56.

3. Thanakone P: Effectivness of intraoral bone block graft in bone augmentation ASEAN plus (2014)34: 1242-1254.

4. Harry D, David $\mathrm{H}$ et al :Alveolar bone grafting and reconstruction procedures prior to implant placement. Dent Clin North Am (2012) 56: 209-218.

5. Precheur HV: Bone graft materials. Dent Clin North Am (2007) 51:729-746.

6. Bellini CM, Romeo D et al: A finite element analysis of tilted versus non-tilted implant configurations in the edentulous maxilla Int J Prosthodont (2009) 22:155-157.

7. Avinash B S, Khalid A: Mini implants for definitive prosthodontic treatment: a systematic review. J Prosthet Dent (2013) 109:156-164.

8. Bertil $\mathrm{F}$ The posterior maxilla: clinical considerations and current concepts using Branemark system implants Periodontology (2008) 47-67-78.

9. Chiriac G, Herten M, Schwarz F, Rothamel D and Beker $\mathrm{J}$ : Autogenous bone chips influence of a new pieozelectric device on chip morphology, cell viability and differentiation J. Clin Periodontal (2005)32(9):994-9.

10. Enneking WF, Eady JL, Burchard EH : Autogenouscortical bone graft in the reconstruction of segmental skeletal defects J bone joint Surg AM(1980)6(7)1039-58.

11. Aghaloo TL, Moy PK. Which hard tissue augmentation techniques are the most successful in furnishing bony support for implant placement. Int $\mathrm{J}$ Oral Maxillofac Implants (2007)22:49-7018.

12. Menini M, Signori A et al : Tilted implants in the immediate loading rehabilitation of the maxilla: a systematic review. J Dent Res (2012) 91(9):821-827

13. Clavero J, Lundgren S : Ramus or Chin Grafts for Maxillary Sinus Inlay and Local Onlay Augmentation: Comparison of Donor Site Morbidity and Complications Clinical Implant Dentistry and Related Research(2003) Volume 5, Number 3, 156-175.
14. Urban I :Vertical and horizontal ridge augmentation Avril du plessis (2017) vol (1)chapter $(3,4)$ 11-28.

15. Khoury F, Antoun H, Missika P, Bessad J, Chiapasco M, Hage G, Happe A, Jakes N et al : Bone Augmentation in oral implantology, Quitentessence Puplishing Co, Ltd (2007) chapter 6:117-219

16. Misch, C.E. Occlusal considerations in implant therapy: clinical guidelines with biomechanical rationale. International Journal of Oral Implantology 6 (2005): 22-31

17. Khoury F, Hanse T: Mandibular bone block harvesting from the retromandibular region: A10 year prospective clinical study, Int J .Oral Maxillofacial implants 201530:688-697.

18. Pikos, Michael A.:"Mandibular block autografts for alveolar ridge augmentation." Atlas of the oral and maxillofacial surgery clinics of North America 13.2 (2005): 91-107

19. Schettler,D. "Sandwich technic with cartilage transplant for raising the alveolar process in the lower jaw." Fortschritte der Kiefer-und Gesichts-Chirurgie 20 (1976):61

20. Park $\mathrm{C}$ and Indersano $\mathrm{T}$, nerve evaluation protocol, California association of maxillofacial surgery jm of maxillofacial surg 44 .431-434; 2014

21. Misch C M: Comparison of Intraoral Donor Sites for Onlay Grafting Prior to Implant Placement INT J oral maxillofac implants 1997;12: 767-776).

22. ACocella A, Bertolair, Colafranceschi M, SaccoR: Clinical, histological and histomorphometric evaluation of the healing of mandibular ramus bone block grafts for alveolar ridge augmentation before implant placement, Journal of Cranio-Maxillo-Facial Surgery (2010) 38, 222e230.

23. Acocella A, Bertolai R, Ellis E, Nissan J, Sacco R : A. Maxillary alveolar ridge reconstruction with monocortical fresh-frozen bone blocks: A clinical, histological and histomorphometric study Journal of Cranio-MaxilloFacial Surgery 40 (2012) 525-533

24. AlGhamdi Ali S: Post-surgical complications of symphyseal block graft with and without soft tissue grafting Saudi Med J 2013; Vol. 34 (6) 609-615.

25. Osman A H. ,Atef M :Computer-guided chin harvest: A novel approach for autogenous block harvest from the mandibular symphesis Clin Implant Dent Relat Res. 2018; $1-6$. 